\title{
On the Membrane Approximation for Thin Elastic Shells in the Hyperbolic Case
}

\author{
E. SánChez-Palencia
}

\begin{abstract}
We consider the variational formulation of the problem of elastic shells in the membrane approximation, when the medium surface is hyperbolic. It appears that the corresponding bilinear form behaves as some kind of two dimensional elasticity without shear rigidity. This amounts to saying that the membrane behaves rather as a net made of elastic strings disposed along the asymptotic curves of the surface than as an elastic two-dimensional medium. The mathematical and physical reasons of this behavior are explained and consequences are thrown conceming the admissible applied forces and the behavior of the solutions. The normal component of the displacement is somewhat non smooth. Our approach gives a description of the problem in somewhat general situations concerning the boundary conditions, whereas the classical approach in terms of a hyperbolic system of total order 4 with 2 double characteristics (the asymptotic lines) only works in the case when the boundary conditions lead to either Cauchy or Goursat problems.
\end{abstract}

\section{INTRODUCTION}

We consider here elastic thin shells in the membrane approximation, when the rigidity with respect to bendings is neglected. Classical references are Goldenveizer [5] and Niordson [8]. This paper is in some sense a continuation of Sánchez-Palencia [12], as well as [9], [10] and [11] where the asympotitics of the shell problem when the thickness tend to zero is considered. It appears that two very different asymptotic processes describe the limit behavior, according to the fact that the middle surface $s$ (along with the corresponding kinematic boundary conditions) admits or no pure

1991 Mathematics Subject Classiffication: 73K15.

Editorial Complutense. Madrid, 1993. 
bendings, i.e. displacements keeping invariant the intrinsic metrics of $s$ (also so-called inextensional displacements). As the rigidity of a thin shell is very much larger to extension (membrane sollicitations) than to bendings, the limit behavior is described by pure bendings provided they are allowed by the surface. Oppositely, if such pure bendings are not allowed, the limit behavior is described by the membrane approximation (along with boundary layers in certain cases). The surfaces which admit pure bendings are called "non inhibited» (or «non rigid» in geometric terminology, but we prefer «non inhibited», as rigidity is a different concept in mechanics). We shall deal here with «inhibited» (or «rigid») surfaces, which lead as we said above, to the membrane approximation.

Let $s$ be a (portion of) surface with boundary $\Gamma$ in the space $\mathbb{R}^{3}$ with coordinates $x=\left(x_{1}, x_{2}, x_{3}\right)$. We shall consider it described by two parameters $y=\left(y^{1}, y^{2}\right)$ running in the domain $\Omega$ of the $y$ plane, so that

$$
x=\varphi(y) ; \varphi: y \in \Omega \rightarrow x \in \mathbb{R}^{3}
$$

and of course the boundary $\delta \Omega$ is mapped onto $\Gamma$. Here and in the sequel the function $\varphi$ will be considered sufficiently smooth and uniformly hyperbolic, i.e. the principal curvatures are of opposite sign and bounded away from zero. At each point of $s$ we consider a local frame formed by the tangent vectors $\mathbf{e}_{\alpha}$ and the unit normal $\mathbf{e}_{3}$ to $s$, where

$$
\mathbf{e}_{\alpha}=\varphi_{, \alpha} \quad \alpha=1,2
$$

and ${ }_{, \alpha}$ denotes differentiation with respect to $y^{\alpha}$. As usually, we shall use greek and latin indices which run in $(1,2)$ and $(1,2,3)$ respectively.

Let $\mathbf{u}=\left(u_{1}, u_{2}, u_{3}\right)$ be the displacement (small, in the linear framework, which will be the only considered here) vector of the points of $s$ when the field of forces $f$ acts upon it. It appears that the simplest formulation is obtained when $\mathbf{u}$ and $\mathbf{f}$ are described in covariant and contravariant components respectively

$$
\begin{aligned}
& \mathbf{u}=\left(u_{1}, u_{2}, u_{3}\right) \\
& \mathbf{f}=\left(f^{1}, f^{2}, f^{3}\right)
\end{aligned}
$$

but other descriptions are possible, and tensor notation will be used. Let $b_{\alpha \beta}$ and $\Gamma_{\alpha \beta}^{\gamma}$ be the second fundamental form and the Christoffel symbols of the surface and let ${ }_{1 \alpha}$ denote covariant differentiation. Then, the strain tensor produced by $\mathbf{u}$, i.e. the variation (in the linearized sense) of the first 
fundamental form of $s$ when it is deformed and $\varphi$ becomes $\varphi+\mathbf{u}$ is given by

$$
\gamma_{\alpha \beta}(\mathbf{u})=\frac{1}{2}\left(u_{\alpha \mid \beta}+u_{\beta \mid \alpha}\right)-b_{\alpha \beta} u_{3}
$$

It will prove useful to define

$$
\tilde{e}_{\alpha \beta}(\mathbf{u})=\frac{1}{2}\left(u_{\alpha \mid \beta}+u_{\beta \mid a}\right)
$$

as well as

$$
e_{\alpha \beta}(\mathbf{u})=\frac{1}{2}\left(u_{\alpha, \beta}+u_{\beta, \alpha}\right)
$$

(which is not a tensor!), so that:

$$
\begin{aligned}
& \gamma_{\alpha \beta}=\tilde{e}_{\alpha \beta}-b_{\alpha \beta} u_{3} \\
& \tilde{e}_{\alpha \beta}=e_{\alpha \beta}-\Gamma_{\alpha \beta}^{\bar{z}} u_{\lambda}
\end{aligned}
$$

We note that $e_{a \beta}$ are the classical expressions of the strain in plane Cartesian coordinates; $\tilde{e}$ involves the Christoffel symbols, accounting for the curvilinear coordinates, and $\gamma$ contains also the normal component $u_{3}$ of the displacement, but not its derivatives. The physical implications of this fact will be discussed later (sect. 5). The study of this paper relies mainly on the elimination of the component $u_{3}$ in order to deal with an operator involving derivatives of the same order for the (remaining) unknowns. The displacement vector will be decomposed into the «reduced displacement» $\hat{\mathbf{u}}$ and $u_{3}$ :

$$
\mathbf{u}=\left(u_{1}, u_{2}, u_{3}\right)=\left(\hat{\mathbf{u}}, u_{3}\right) ; \quad \hat{\mathbf{u}}=\left(u_{1}, u_{2}\right)
$$

We note that $\gamma$ depend on $\mathbf{u}$, but $e$ and $\tilde{e}$ depend only on $\hat{\mathbf{u}}$.

Let $a^{\alpha \beta \lambda \mu}$ be the elasticity coefficients of the membrane, written in contravariant components associated with the frame $\mathbf{e}_{\alpha}$. They are given functions on $\Omega$ under very general hypotheses, including anisotropic elasticity. They satisfy the symmetry and positivity conditions all over $\Omega$ :

$$
\begin{gathered}
a^{\alpha \beta \lambda \mu \mu}=a^{\beta \alpha \lambda \mu}=a^{\lambda_{\mu \alpha} \alpha \beta} \\
a^{\alpha \beta \lambda \mu} e_{\lambda \mu} e_{\alpha \beta} \geq c e_{\alpha \beta} e_{\alpha \beta} \quad \forall \text { symmetric } e_{\alpha \beta}
\end{gathered}
$$


where $c$ denote some positive constant.

Let $T^{a \beta}$ denote the contravariant components of the stress tensor:

$$
T^{a \beta}=a^{\alpha \beta \lambda \mu} \gamma_{\lambda \mu}(\mathbf{u})
$$

Then the equations for the membrane approximation are:

$$
\left\{\begin{array}{l}
-T_{\mid \alpha}^{\alpha \beta}=f^{\beta} \\
-b_{\alpha \beta}^{\alpha \beta} T^{\alpha \beta}
\end{array}\right.
$$

the two first equations (1.14) for $\beta=1,2$ are analogous to the equations of two-dimensional elasticity, but they involve also $u_{3}$. The third one is the normal component of the equilibrium equation.

In a functional space $\overline{\bar{V}}$ which will be specified later, the variational formulation of (1.13), (1.14) is:

$$
\left\{\begin{array}{l}
\text { Find } \mathbf{u} \in \overline{\bar{V}} \text { such that } \\
a(\mathbf{u}, \mathbf{v})=<\mathbf{f}, \mathbf{v}>
\end{array} \quad \mathbf{v} \in \overline{\bar{V}}\right.
$$

where $a$ is the bilinear and symmetric form

$$
\begin{gathered}
a(\mathbf{u}, \mathbf{v})=\int_{s} a^{\alpha \beta \lambda \mu} \gamma_{\mu \mu}(\mathbf{u}) \gamma_{\alpha \beta}(\mathbf{v}) d s \\
<\mathbf{f}, \mathbf{v}>=\int_{s} f^{i} v_{i} d s
\end{gathered}
$$

In order to define the space $\overline{\bar{V}}$, we shall recall some features of [12]. The membrane problem is the asymptotic form of another problem with rigidity with respect to bendings, which involve fourth order derivatives of $u_{3}$. This complete problem is worked out in a space $V$ of kinematically admissible functions

$$
V=\left\{\mathbf{u}=\left(u_{1}, u_{2}, u_{3}\right) \in H^{\prime}(s) \times H^{1}(s) \times H^{2}(s), \text { bound. cond. }\right\}
$$

where «bound. cond.» means that $\mathbf{u}$ must satisfy the kinematic boundary conditions of the «complete problem» (see Bernadou and Ciarlet [1] and Ciarlet and Miara [3] for these questions. The shell (or surface) is said to be inhibited if $\mathbf{u} \in V$ and $\gamma_{\alpha \beta}(\mathbf{u})=0$ implies $\mathbf{u}=0$. In this case, $a(\mathbf{v}, \mathbf{v})^{1 / 2}$ is a norm in $V$, and $\overline{\bar{V}}$ is the completion of $V$ for this norm. This is the natural space for the study of the membrane approximation, which is con- 
sidered in (1.15). As for the right side of (1.15), it is given by (1.17) or more generally it denotes the duality between the dual of $\overline{\bar{V}}$ (denoted by $\overline{\bar{V}}^{\prime}$ ) and $\overline{\bar{V}}$.

It is clear that some of the boundary conditions of (1.18) are lost by passing to $\overline{\bar{V}}$. This is the classical situation in singular perturbation theory (as the fourth order derivatives of $u_{3}$ in the complete problem disappear in the membrane approximation). These lost boundary conditions are of course associated with boundary layers in the asymptotic process. The specific boundary conditions for $\overline{\bar{V}}$ depend on the physical problem. We shall give in section 3 some examples with specific description of the boundary conditions. The sake of this paper is to investigate the structure of the problem (1.15) in the case of a hyperbolic surface. A formal reduction to a problem for $\hat{\mathbf{u}}=\left(u_{1}, u_{2}\right)$ will be done in the next section. The study of the structure of the solutions and of the admissible forces $f$ for the membrane problem is then done in sections 4 and 6 . It exhibites a clear weakness of the membrane in the direction of the asymptotic lines of the surface.

\section{ELIMINATION OF $u_{3}$. THE REDUCED PROBLEM}

As the surface is uniformly hyperbolic, the asymptotic lines are well defined at every point and we may choose the parameters $y^{1}, y^{2}$ in such a way that the asymptotic lines are the "coordinate lines" $y^{\prime}=$ const., $y^{2}=$ const. In this case, the second fundamental form is such that

$$
b_{11}=b_{22}=0, \quad b_{12} \neq 0
$$

where $b_{12}$ is a smooth function bounded away from zero. Then only the component $\gamma_{12}(\mathbf{u})$ (the «shear" component) contains $u_{3}$ :

$$
\left\{\begin{array}{l}
\gamma_{11}(\mathbf{u})=\tilde{e}_{11}(\hat{\mathbf{u}})=u_{1 \mid 1} \\
\gamma_{22}(\mathbf{u})=\tilde{e}_{22}(\hat{\mathbf{u}})=u_{2 \mid 2} \\
\gamma_{12}(\mathbf{u})=\tilde{e}_{12}(\hat{\mathbf{u}})-b_{12} u_{3}=\frac{1}{2}\left(u_{1 \mid 2}+u_{2 \mid 1}\right)-b_{12} u_{3}
\end{array}\right.
$$

then, taking successively the test function in (1.15) $v$ of the forms

$$
\begin{gathered}
\mathbf{v}=\left(0,0, v_{3}\right) \\
\mathbf{v}=\left(v_{1}, v_{2}, 0\right)=\hat{\mathbf{v}}
\end{gathered}
$$

we have 


$$
\begin{gathered}
\int_{s} 2 a^{12 \lambda \mu} \gamma_{\lambda \mu}(\mathbf{u})\left(-b_{12} v_{3}\right) d s=\int_{s} f^{3} v_{3} d s \\
\int_{s} a^{\alpha \beta \lambda \mu} \gamma_{\lambda \mu}(\mathbf{u}) \tilde{e}_{\alpha \beta}(\hat{\mathbf{v}}) d s=\int_{s} f^{\alpha} v_{a} d s
\end{gathered}
$$

As we may take in (2.5) for instance $v_{3} \in \mathscr{Q}(s)$ we have

$$
-2 b_{12} a^{12 \lambda \mu} \gamma_{\lambda \mu}(\mathbf{u})=f^{3}
$$

which by virtue of (2.2) becomes

$$
-2 b_{12} a^{12 \lambda \mu} \tilde{e}_{\lambda \mu}(\hat{\mathbf{u}})+4\left(b_{12}\right)^{2} a^{1212} u_{3}=f^{3}
$$

Now, as $b_{12}$ is different from zero, using the hypothesis of positivity (1.12), we see that the coefficient of $u_{3}$ in (2.8) never vanishes, and (2.8) allows us to write $u_{3}$ in terms of $\hat{\mathbf{u}}$ :

$$
u_{3}=\left[4\left(b_{12}\right)^{2} a^{1212}\right]^{-1}\left[2 b_{12} a^{12 \lambda \mu} \bar{e}^{\lambda \mu \mu}(\hat{\mathbf{u}})+f^{3}\right]
$$

which we replace into (2.6), which become

$$
\hat{a}(\hat{\mathbf{u}}, \hat{\mathbf{v}})=\Phi(\hat{\mathbf{v}})
$$

where

$$
\begin{gathered}
\hat{a}(\hat{\mathbf{u}}, \hat{\mathbf{v}})=\int_{s} \hat{a}^{\alpha \beta \lambda \mu} \bar{e}_{\lambda \mu}(\hat{\mathbf{u}}) \vec{e}_{\alpha \beta}(\hat{\mathbf{v}}) d s \\
\hat{a}^{\alpha \beta \lambda \mu}=a^{\alpha \beta \lambda \mu}-\frac{a^{\alpha \beta 12} a^{12 \lambda \mu \mu}}{a^{1212}} \\
\Phi(\hat{\mathbf{v}})=\int_{S}\left[f^{\alpha} v_{\alpha}+\frac{a^{\alpha \beta 12} f^{3}}{2 b_{12} a^{1212}} \overline{\mathbf{e}}_{\alpha \beta}(\hat{\mathbf{v}})\right] d s
\end{gathered}
$$

Then, problem (1.15) reduces to problem (2.10) in an appropriate space, $u_{3}$ being the calculated expression (2.9). Problem (2.10) will be called the reduced problem. We shall formulate it more precisely in Theorem 6.4. It recalls an elasticity two-dimensional problem; but in fact it is very diferent from two dimensional elasticity. Indead, it is apparent from (2.12) that $\hat{a}^{1212}=0$ and consequently the reduced coefficients will not satisfy an inequality analogous to (1.12). We shall see that it amounts to some kind of two-dimensional elasticity without rigidity with respect to 
shear (i.e. to $\tilde{e}_{12}$, in the special coordinates where the asymptotic lines are the coordinate curves).

The properties of the reduced coefficients (at a fixed point of $s$ ) are given by:

Theorem 2.1. The reduced coefficients $\hat{a}^{a \beta i z}$ satisfy the symmetries:

$$
\hat{a}^{\alpha \beta \lambda \mu}=\hat{a}^{\beta \omega \lambda \mu}=\hat{a}^{i \mu \alpha \beta} .
$$

There exist $c_{1}>0$ and $c_{2}>0$ such that

$$
c_{1}\left(\tilde{e}_{11}^{2}+\tilde{e}_{22}^{2}\right) \leqslant \hat{a}^{\alpha \beta \lambda \mu} \tilde{e}_{\lambda \mu} \tilde{e}_{\alpha \beta} \leqslant c_{2}\left(\tilde{e}_{11}^{2}+\tilde{e}_{22}^{2}\right) \quad \forall \text { symmetric } \tilde{e}_{\alpha \beta} .
$$

Clearly, the form involved in (2.15) is a bilinear form on the space of the second order symmetric tensors, which is isomorphic to $\mathbb{R}^{3}$ (components $\tilde{e}_{11}, \tilde{e}_{22}, \tilde{e}_{12}=\tilde{e}_{21}$ ).

Proof of theorem 2.1. The symmetries are evident from (1.11) and (2.12). Let us study the bilinear form appearing in (2.15). Taking $\bar{e}_{12}=\bar{e}_{21}$, other $\tilde{e}_{\alpha \beta}=0$, its value is $4 \hat{a}^{1212}\left(\bar{e}_{12}\right)^{2}=0$ by (2.12). We are now proving that this is the only $\tilde{e}$ where the form vanishes. Let $\tilde{e}_{a \beta}$ be symmetric and such that

$$
\hat{a}^{\alpha \beta \lambda \mu} \tilde{e}_{\lambda \mu \alpha} \tilde{e}_{\alpha \beta}=0 .
$$

Let us define the number

$$
u_{3}=a^{12 \lambda \mu} \tilde{e}_{\lambda \mu}\left(2 a^{1212} b_{12}\right)^{-1}
$$

with the considered $\bar{e}_{\lambda \mu}$. As the only $b_{a \beta}$ different from zero is $b_{12}$, we have

$$
a^{12 \lambda \mu}\left(\tilde{e}_{\lambda \mu}-b_{j \mu} u_{3}\right)=0 .
$$

We then consider the numbers

$$
\gamma_{\lambda \mu}=\bar{e}_{\lambda \mu}-b_{\lambda \mu} u_{3}
$$

and (2.18) becomes 


$$
a^{12 \lambda \mu} \gamma_{i \mu}=0 .
$$

Multiplying by $-b_{12} u_{3}$ and as the only $b_{a \beta}$ different from zero is $b_{12}$, we have

$$
-2 a^{12 \lambda \mu} \gamma_{\lambda \mu} b_{12} u_{3}=0
$$

and

$$
-a^{\alpha \beta \lambda \mu} \gamma_{\lambda \mu} b_{\alpha \beta} u_{3}=0 .
$$

On the other hand, (2.16) on account of (2.12) writes:

$$
a^{a \beta \lambda \mu}\left[\tilde{e}_{\lambda \mu}-b_{\lambda \mu} \frac{2 a^{12 \gamma \delta}-\bar{e}_{\alpha \phi}}{4 a^{1212} b_{12}}\right] \bar{e}_{\alpha \beta}=0
$$

where again was used the fact that the only $b_{a \beta}$ different from zero is $b_{12}$. But (2.22) amounts to

$$
a^{\alpha \beta \lambda \mu} \gamma_{\lambda \mu} \tilde{e}_{\alpha \beta}=0
$$

Adding (2.21) and (2.23) we have

$$
a^{\alpha \beta \lambda \mu} \gamma_{\lambda \mu} \gamma_{\alpha \beta}=0
$$

which, on account of the positivity (1.12) of the coefficients, give

$$
\left\{\begin{array}{l}
0=\gamma_{11}=\bar{e}_{12} \\
0=\gamma_{22}=\bar{e}_{22} \\
0=\gamma_{12}=\bar{e}_{12}-b_{12} u_{3}
\end{array}\right.
$$

and consequently the only $\tilde{e}_{\alpha \beta}$ different from zero is $\tilde{e}_{12}$.

Let us prove now that

$$
\hat{a}^{\alpha \beta i \lambda} \tilde{e}_{j \mu} \tilde{e}_{\alpha \beta} \geqslant 0 \quad \forall \text { symmetric } \tilde{e}_{a \beta}
$$

We proceed as before from (2.16), which is not necessarily equal to zero. Using (2.17) we get

$$
\hat{a}^{\alpha \beta \lambda \mu} \dot{e}_{i \mu \mu} \tilde{e}_{\alpha \beta}=a^{\alpha \beta \lambda \mu} \gamma_{\mu \mu} \gamma_{\alpha \beta}
$$

which is $\geqslant 0$ by virtue of (1.12), and (2.26) is proven. 
Now, the form $\hat{a}^{\alpha \beta \lambda \mu} \tilde{e}_{\lambda \mu} \bar{e}_{\alpha \beta}$ is symmetric and then diagonable. Their eigenvalues are $\geqslant 0$. As it only vanishes on the subspace $\tilde{e}_{11}=\tilde{e}_{22}=0$, $\tilde{e}_{12} \neq 0$, zero is a simple eigenvalue. The two other eigenvalues are greater than zero, and the corresponding eigenvectors are orthogonal to $\left(0,0, \tilde{e}_{12}\right)$. They form a basis of the subspace $\left(\tilde{e}_{11}, \tilde{e}_{22}, 0\right)$ where the form is positive definite, and we have (2.15).

\section{MODEL PROBLEMS}

The developments of the following sections depend strongly on the geometric disposition of the characterisitcs (asymptotic curves of $s$ ) and the corresponding boundary conditions. A first condition is that $s$ is a «inhibited surface» which amounts to saying that the rigidity system

$$
\gamma_{\alpha \beta}(\mathbf{u})=0
$$

with the boundary conditions ensures uniqueness $(u=0)$. Taking as in section 2 as coordinate lines the asymptotic curves, we have (2.1), and (3.1) is equivalent to

$$
\begin{gathered}
u_{1 \mid 1}=0, \quad u_{2 \mid 2}=0 \\
\frac{1}{2}\left(u_{1 \mid 2}+u_{2 \mid 1}\right)-b_{12} u_{3}=0
\end{gathered}
$$

and in fact inhibition (or uniqueness) only concerns the hyperbolic system of two equations (3.2); $u_{1}=u_{2}=0$ implies $u_{3}=0$ because of (2.1). Then, in order to have the uniqueness, it is sufficient to have either

$$
u_{1}=u_{2}=0
$$

on a non-characteristic curve (Cauchy problem) or

$$
\begin{cases}u_{1}=0 & \text { on } y^{1}=\text { const. } \\ u_{2}=0 & \text { on } y^{2}=\text { const. }\end{cases}
$$

(Goursat problem) in such a way that $\Omega$ is contained in the determinacy domain of the curves bearing the boundary conditions. We now specify some «model examples» which will be considered in the sequel.

In the following figures, the curvilinear coordinates are of course the special ones, and $y^{1}=$ const., $y^{2}=$ const. are the two families of characteristics. 
Example 3.1. The domain $\Omega$ has a smooth boundary $\partial \Omega$ which is noncharacteristic unless at some isolated points (fig. 3.1). The surface is fixed along the whole boundary, (i.e. (3.4) is prescribed there).

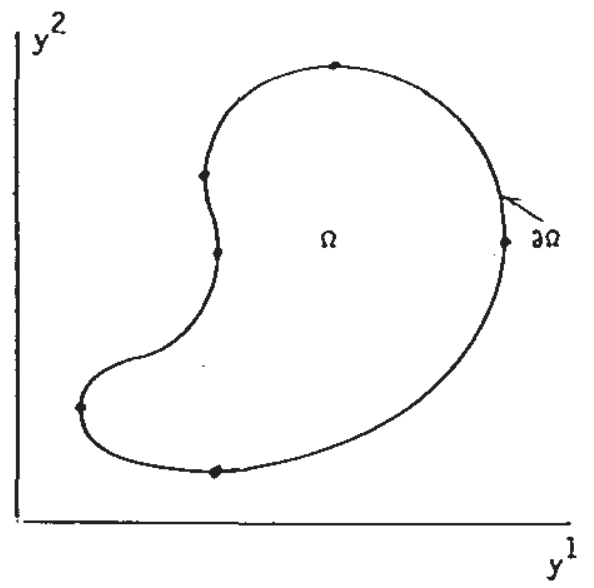

Figure 3.1

Example 3.2. Analogous to the previous one, but the part $\Gamma_{\mathrm{t}}$ of the boundary is free (there are no kinematic boundary condition on $\Gamma_{1}$ and (3.4) is prescribed on $\Gamma_{0}$ ). Of course the characteristics issued from any point of $\Gamma_{1}$ cannot go out of $\Omega$ before cutting $\Gamma_{0}$ (fig. 3.2).

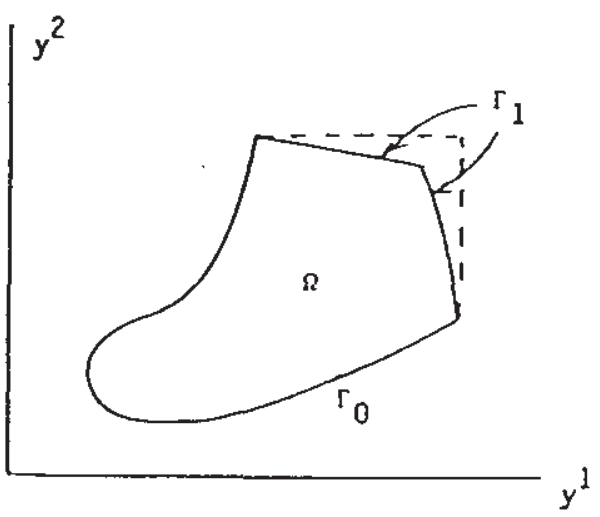

Figure 3.2 
Example 3.3. Analogous to the previous one, but $\Gamma_{0}$ is formed by two characteristics. (3.5) is prescribed on them (fig. 3.3).

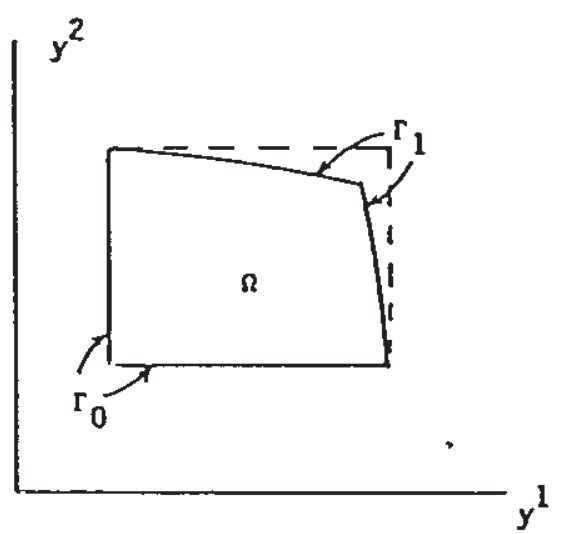

Figure 3.3

Example 3.4. A portion of a hyperbolic hyperboloid as shown in fig. 3.4.a. The corresponding $\Omega$ is shown in fig. 3.4.b where the lines $A B$ and $A^{\prime} B^{\prime}$ are identified. The surface is fixed by $\Gamma_{0}$ and $\Gamma_{1}$ (boundary conditions (3.4) on both).

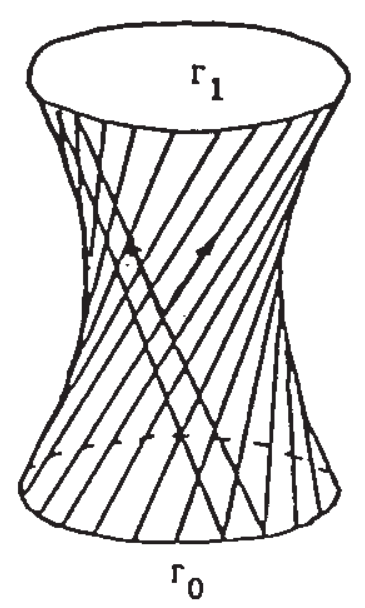

Figure 3.4.a.

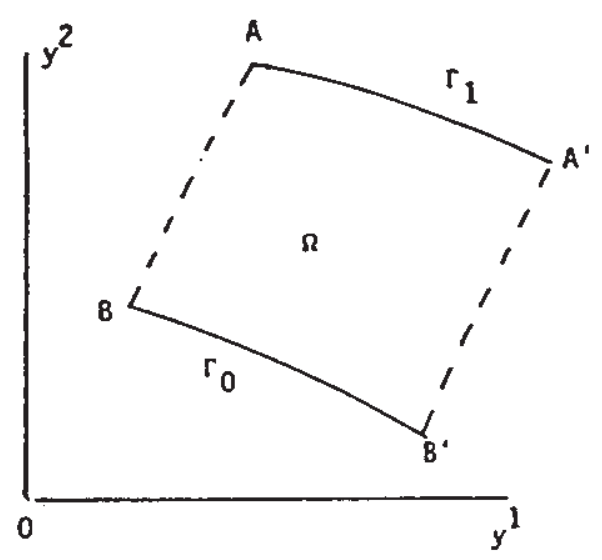

Figure 3.4.b. 
Example 3.5. The same as the previous one, but fixed on $\Gamma_{0}$ and free on $\Gamma_{1}$ (i.e. (3.4) on $\Gamma_{0}$, and no boundary conditions on $\Gamma_{1}$ ).

\section{THE REDUCED BILINEAR FORM AND THE ASSOCIATED ENERGY SPACE}

Let us consider the reduced bilinear form $\hat{a}$ defined by $(2.11),(2.12)$ in the case of the model examples of section 3 . We are considering the corresponding energy space $\hat{V}$ (the classical space associated with the reduced problem (2.10)). This space will be the completion with respect to the norm defined by the form $\hat{a}^{1 / 2}$ of the space

$$
\dot{V}=\left\{\hat{\mathrm{v}} \in H^{\prime} \times H^{1} ; \quad \text { boundary conditions }\right\}
$$

where «boundary conditions» means that $\hat{\mathbf{v}}$ must satisfy the kinematic boundary conditions specified in the examples of sect. 3 . This definition of $V$ is clearly issued from (1.18), taking only the tangential components. The completion process follows from the singular perturbation associated with neglecting the rigidity with respect to bendings.

Using the general hypotheses of uniformity of the geometric properties of $s$ mentionned in section 1, we deduce of theorem 2.1:

Theorem 4.1. The form $\hat{a}(\hat{\mathbf{u}}, \hat{\mathbf{v}})$ is symmetric and satisfies the estimates:

$$
c_{1} \int_{s}\left(\tilde{e}_{11}^{2}+\tilde{e}_{22}^{2}\right) d s \leqslant \hat{a}(\hat{\mathbf{v}}, \hat{\mathbf{v}}) \leqslant c_{2} \int_{s}\left(\left(\dot{e}_{11}^{2}+\tilde{e}_{22}^{2}\right) d s\right.
$$

where $\bar{e}_{11}$ and $\bar{e}_{22}$ are the expression corresponding to (1.9), (1.7) for the considered $\hat{\mathbf{v}}$, and $c_{1}, c_{2}$ are positive constants independent of $\hat{\mathbf{v}}$.

Now ket us consider the completion process from $\dot{V}$ to $\hat{V}$ for the norm $\hat{a}^{\prime 2}$. Obviously if

$$
\dot{V} \ni \hat{\mathbf{u}}^{j} \rightarrow \hat{\mathbf{u}} \quad \text { in } \hat{V}
$$

then we have from (4.2): 


$$
\left\{\begin{array}{l}
\partial_{1} u_{1}^{j}-\Gamma_{11}^{\alpha} u_{\alpha}^{j}=\tilde{e}_{11}^{j} \rightarrow \tilde{e}_{11}=\partial_{1} u_{1}-\Gamma_{11}^{\alpha} u_{\alpha} \\
\partial_{2} u_{2}^{j}-\Gamma_{22}^{\alpha} u_{\alpha}^{j}=\tilde{e}_{22}^{j} \rightarrow \tilde{e}_{22}=\partial_{2} u_{2}-\Gamma_{22}^{\alpha} u_{\alpha}
\end{array}\right.
$$

with convergence in $L^{2}$. But (4.4) is a hyperbolic system for $\hat{\mathbf{u}}^{j}$ (or $\hat{\mathbf{u}}$ in the limit), which also satisfy either Cauchy or Goursat boundary conditions in all examples of section 3 . According to general features of hyperbolic systems (cf. for instance Courant Hilbert [4]) this implies $L^{2}$ convergence. Moreover, as the system is weakly coupled, $L^{2}$ convergence of $\hat{\mathbf{u}}$ and of the right hand sides implies also $L^{2}$ convergence of $\partial_{1} u_{1}$ and $\partial_{2} u_{2}$. Then, we have proved

Lemma 4.2. In the case of the model examples of section 3, we have:

$$
\hat{\nabla} \subset\left\{\hat{\mathbf{v}} \in\left(L^{2}(\Omega)\right)^{2}, \quad \partial_{1} v_{1} \in L^{2}(\Omega), \quad \partial_{2} v_{2} \in L^{2}(\Omega)\right\}
$$

The classical properties of traces in $L^{2}$ (see for instance Smirnov [13], sect. 113) then hold for $u_{1}$ (resp. $u_{2}$ ) on curves which are not tangent to the characteristics $y^{2}=$ const. (resp. $y^{1}=$ const.). Of course, traces of $u_{1}$ (resp. $u_{2}$ ) tangent to the corresponding characteristics do not make sense, as $\partial_{2} u_{1}$ and $\partial_{1} u_{2}$ do not belong to $L^{2}$; then in the completion process the kinematic boundary conditions which make sense according to the previous considerations, are stable and are satisfied by any element of $\hat{V}$. The other boundary conditions are clearly lost by completion; indeed, the space $\hat{V}$ is essentially analogous, concerning $u_{1}$, to

$$
L_{y^{2}}^{2}\left(0, a ; H_{y^{1}}^{1}(0, b)\right)
$$

and traces on $y^{2}=$ const. desappear by completion (see for instance Bourbaki [2], p. 133, Proposition 10). Finally we have:

Theorem 4.3. Under the general uniformity hypotheses of section 1, in the model examples of section 3, we have:

$$
\hat{V} \subset\left\{\hat{\mathrm{v}} \in\left(L^{2}\right)^{2}, \quad \partial_{1} v_{1} \in L^{2}, \quad \partial_{2} v_{2} \in L^{2}, \quad \text { bound. cond. }\right\}
$$

where $y^{1}, y^{2}$ are the special curvilinear coordinates associated with the asymptotic curves of s. Moreover, the boundary conditions in (4.7) are, in each model example, those for $u_{1}$ and $u_{2}$ which are along curves which are transversal to $y^{2}=$ const. and $y^{1}=$ const. respectively. 
Remark 4.4. In most of the model examples we specified boundary conditions which are stable by completion. For instance, in example 3.3 we specified (3.5) which fulfils the transversality conditions. Of course we may consider slight modification of the examples, for instance, prescribing $u_{1}=u_{2}=0$ on $\Gamma_{0}$ of example 3.3 , at the level of the non-complete space $\dot{V}$ (4.1), but, in the completion process, passing to $\hat{V}$ we only keep (3.5). On the other hand, the trace properties are not uniform in cases as example 3.1 , as the trace of $u_{1}$ and $u_{2}$ does not make sense at points with horizontal and vertical tangent, respectively. These are classical features of spaces of functions with certain (not all) partial derivatives belonging to $L^{2}$ and we will not insist on them.

Remark 4.5. Obviously Lemma 4.2 implies that $\hat{V}$ is contained in $L^{2}$. This only concerns the tangential components $u_{1}, u_{2}$, not $u_{3}$, and this do not implies that, in the framework of Sánchez-Palencia [12], $\overline{\bar{V}} \subset H$, which is generally false in the case considered here.

\section{PHYSICAL CONSIDERATIONS}

We saw in sections 2 and 4 that the reduced problem behaves as some kind of two-dimensional elasticity without shear rigidity, as $\bar{e}_{12}$ lacks in $(2,15)$.

Of course we are speaking of shear in curvilinear coordinates, when the coordinate lines coincide with the asymptotic curves of the surface. This behavior is analogous to that of a net with filaments disposed along the asymptotic curves. This peculiar behavior is due of course to the $u_{3}$ component of the displacement, which allows the surface to go out of itself in the deformation process. We are showing with a heuristic reasoning that a shear deformation, accompanied by a convenient $u_{3} \neq 0$ may be performed with elastic stresses $T^{\alpha \beta}=0$. 


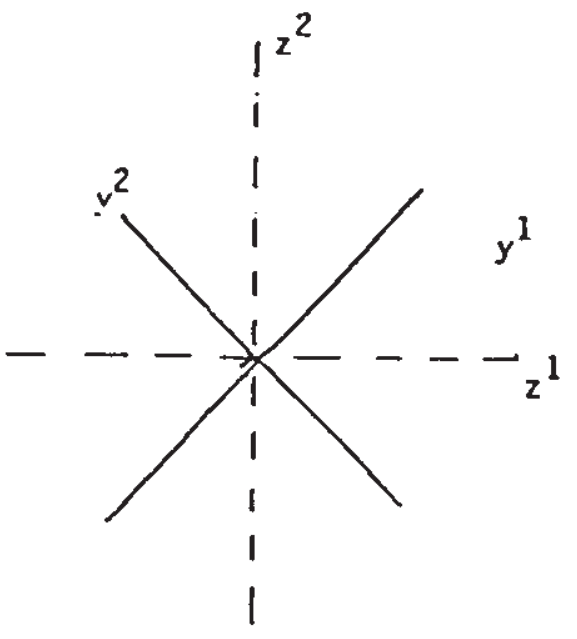

Figure 5.1.a.

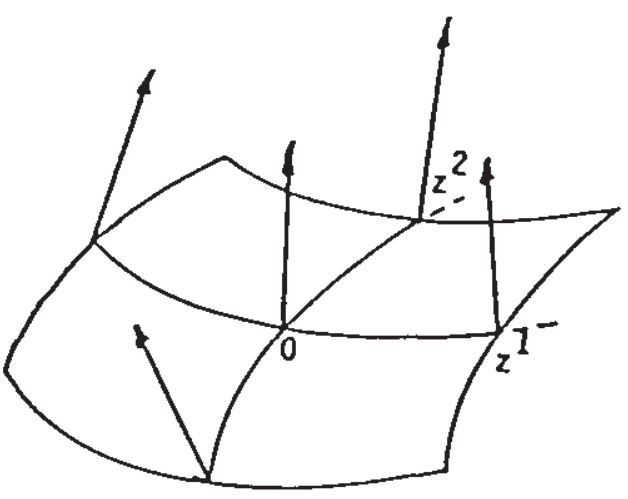

Figure 5.1.b.

Let us consider, to fix ideas, a point where the asymptotic curves are orthogonal. It is known that a shear in the directions of the axes $y^{1}, y^{2}$ is equivalent to an allongement and a compression in the axes $z^{1}, z^{2}$ in the direction of the bissectings, which are here the lines of curvature (fig. 5.1.a). A sketch of the surface in a neighbourhood of 0 with the corresponding normals is given in fig. 5.1.b. It is then aparent that, giving a positive $u_{3}$ and vanishing $u_{1}, u_{2}$, we will have an allongation and a shortening in the directions of $z^{2}$ and $z^{1}$ respectively. Then giving appropriate $\tilde{e}_{11}$ and $\tilde{e}_{22}$ (positive and negative) we will have a vanishing deformation of the element of surface, and consequently vanishing stresses $T^{\alpha \beta}$ of the membrane.

We may also point out that the proof of theorem 2.1 involves some of these facts, and in (2.25) is exhibited a state of vanishing strain $\gamma$ (and them vanishing stress $T^{\alpha \beta}$ ) with non-vanishing $\tilde{e}_{\alpha \beta}$ because of the term $u_{3}$.

\section{THE ADMISSIBLE FORCES AND THE NON-SMOOTHNESS OF THE SOLUTION}

In section 4 we considered the structure of the form $\hat{a}$ in $(2.10)$. We are now considering the linear form $\Phi$ on the right hand side $(2.10)$ and the corresponding forces $f$ in order to define a continuous form on $\hat{v}$. Using (2.2), the functional (2.13) becomes 


$$
\Phi(\hat{\mathbf{v}})=\Phi_{1}+\Phi_{2}
$$

with

$$
\begin{aligned}
& \Phi_{1} \equiv \int_{s}\left[f^{\alpha}-\frac{a^{\gamma \delta / 2} f^{3}}{2 b_{12} a^{1212}} \Gamma_{\gamma \delta}^{\alpha}\right] v_{\alpha} d s \\
& \Phi_{2} \equiv \int_{s} \frac{a^{\alpha \beta / 2} f^{3}}{4 b_{12} a^{1212}}\left(\partial_{\alpha} v_{\beta}+\partial_{\beta} v_{\alpha}\right) d s
\end{aligned}
$$

Let us examine successively these two terms, which must define continuous functionals on $\hat{V}$.

Let us consider $\Phi_{1}$. The brackets in (6.2) must merely define an element of the dual $\hat{V}^{\prime}$ of $\hat{V}$. Of course, we may take the functions in the brackets of class $L^{2}$ but we also may take them in larger spaces. Roughly speaking according to (4.6), we may take the bracket in a space of functions of class $L^{2}$ of the variable $y^{2}$ with values in the dual of $H^{1}$ of the variable $y^{1}$, (on account of course of the kinetic boundary conditions prescribed on the last). To be a little more precise, as the sections by $y^{2}=$ const. are in general intervals of the variable $y^{1}$ which depend on $y^{2}$ (see the examples), the space of the $v_{1}$ may be described in terms of measurable sums of spaces, as in Lions [7], p. 62

$$
v_{1} \in \int H_{y^{1}}^{1}\left(y^{2}\right) d y^{2}
$$

and the bracket' may be taken in its dual:

$$
\int H_{y^{1}}^{1}\left(y^{2}\right)^{\prime} d y^{2}
$$

(on account of the kinematic boundary conditions, of course).

Taking $f^{3}=0, f^{4}$ in the space (6.4) (and $f^{2}$ in the corresponding analogous space), and applying the Lax-Milgram theorem to (2.10) we see that the corresponding operators defined are isomorphisms between $\hat{V}$ and its dual; consequently the corresponding solutions fill the space $\hat{V}$.

Let us consider now $\Phi_{2}$. Let us define

$$
\tilde{f}^{3}=\frac{a^{\alpha \beta 12}}{4 b_{12} a^{1212}} .
$$


As this function is multiplied by the derivatives of $\hat{v}$, which does not belong to $L^{2}$ in general, it must be a rather smooth function in order to define a continuous functional. For the time being, we shall take it of class $C^{\infty}$ on $\bar{\Omega}$, and we shall consider later other less smooth functions passing to the limit. In the same way, $\hat{\mathbf{v}}$ in (6.3) will be considered smooth, before passing to the limit in $\hat{V}$. Integrating by parts (on $\Omega$, not on $s$ ), we have

$$
\begin{gathered}
\Phi_{2}=\int_{S} \tilde{f}^{3} v_{1,2} d s=\int_{\Omega} \tilde{f} \frac{d s}{d y} v_{1.2} d y= \\
=\int_{\partial \Omega} \tilde{f}^{3} \frac{d s}{d y} v_{1} v_{2} d l-\int_{\Omega}\left(\tilde{f}^{3} \frac{d s}{d y}\right)_{, 2} v_{1} d y
\end{gathered}
$$

and on analogous expression for $v_{2.1}$. Here $v_{\alpha}$ are the components of the unit outer normal to $\Omega$ in its plane. Let us now pass to the limit for any $\hat{v} \in \hat{V}$, with a smooth $\hat{f}^{3}$. The integrals on $\Omega$ give a bounded functional on $\hat{V}$. The same is true for the line integrals on the portions of $\partial \Omega$ where the traces of $v_{1}$ make sense. Oppositely the integrals on the portions of the boundary where the trace of $v_{1}$ does not make sense (boundaries with $y^{2}=$ const.) define an unbounded functional on $\hat{V}$. As $v_{2}$ does not vanish there, we must have $\tilde{f}^{3}$ equal to zero on such boundaries. Under this condition, we may pass to the limit in $\tilde{f}^{3}$, and we have

Lemma 6.1. The function $f^{3} \in H^{1}(\Omega)$ with vanishing traces on the boundaries along the asymptotic curves define a continuous functional $\Phi_{2}$ in (6.3).

Remark 6.2. We shall not consider the largest class of functions $f^{3}$ such that $\Phi_{2}$ be a bounded functional on $\hat{V}$. Nevertheless, it is easily seen that a function $f^{3}$ which is piecewise smooth and that have a discontinuity along a smooth curve (either characteristic or transversal to the characteristics) give an unbounded functional.

Finally we have:

Theorem 6.3. If $f^{1}$ is chosen in the space (6.4), $f^{2}$ in the analogous space with permutation of the variables, and $f^{3}$ according to Lemma 6.1, then $\Phi$ in (6.1) is a bounded functional on the space $\hat{V}$. This is the case in particular, if $f^{\alpha} \in \mathrm{L}^{2}(\Omega)(\alpha=1,2)$ and $f^{3} \in H^{1}(\Omega)$ and its trace vanish on the parts of the boundary which coincide with asymptotic curves. 
We have now at our disposal elements for the solution of problem (2.10) in the classical framework of the Lax-Milgram theorem.

Theorem 6.4. Let $f$ be chosen according to theorem 6.3. Then the problem

Find $\hat{\mathbf{u}} \in \hat{V}$ such that

$$
\hat{a}(\hat{\boldsymbol{u}}, \hat{\mathbf{v}})=\Phi(\hat{\mathbf{v}}) \quad \forall \hat{v} \in \hat{V}
$$

has a unique solution. Moreover, the set of solutions $\hat{\mathbf{u}}$ fills the space $\hat{V}$ when $\boldsymbol{f}$ fill the space of theorem 6.3; this is even true with $f_{3}=0$.

It is clear that solution is understood in theorem 6.4 in the sense of the reduced vector $\hat{\mathbf{u}}=\left(u^{1}, u^{2}\right) \in \hat{V}$. The normal component $u_{3}$ is then defined by (2.9). We are now showing that $u_{3}$ is in general a non-smooth function, and even a distribution.

As an example, let us consider f such that $u_{1}$ is a piecewise smooth function discontinuous along $y^{2}=0$, the jump being $\varphi\left(y^{\prime}\right)$, smooth function of $y^{1}$. Moreover, let $u_{2}=0$. This is of course possible, for instance with appropriate $f^{1}, f^{2}$ and $f^{3}=0$. Then, (2.9) gives

$$
u_{3}=\frac{a^{12 a \beta}}{2 b_{12} a^{1212}} \tilde{e}_{a \beta}(\hat{\mathbf{u}})=\frac{\varphi\left(y^{1}\right)}{4 b_{12}} \delta\left(y^{2}\right)+\text { terms in } L^{2}(\Omega)
$$

\section{COMPARISON WITH THE HYPERBOLIC SYSTEM AND CONCLUDING REMARKS}

We are comparing our method with the classical treatment of system (1.13) (1.14), which may be reduced to a hyperbolic system of 4 equations of first order with double characteristics (the asymptotic curves of $s$ ).

Let us take again the parameters $y^{1}, y^{2}$ associated with the asymptotic curves. Then, the last equation (1.14) gives

$$
T^{12}=-\left(2 b_{12}\right)^{-1} f^{3}
$$

and $T^{12}$ is known. The other two equations (1.14) then become:

$$
\left\{\begin{array}{l}
T_{.1}^{11}=L_{1}\left(T^{11}, T^{22}, y\right) \\
T_{.2}^{22}=L_{2}\left(T^{11}, T^{22}, y\right)
\end{array}\right.
$$


where $L_{1}, L_{2}$ are functions of their arguments linear in $T^{11}, T^{22}$. This is clearly a hyperbolic system of first order for $T^{11}, T^{22}$, with characteristics the asymptotic curves. Moreover, inverting the matrix $a^{\alpha \beta \gamma d}$, which is possible because of (1.12), (1.13) becomes

$$
\gamma_{\alpha \beta}=b_{\alpha \beta \lambda \mu} T^{\lambda \mu}
$$

and in particular $\gamma_{11}$ and $\gamma_{22}$ give, with (2.2):

$$
\left\{\begin{array}{l}
u_{1,1}=L_{3}\left(u_{1}, u_{2}, T^{11}, T^{22}, y\right) \\
u_{2,2}=L_{4}\left(u_{1}, u_{2}, T^{11}, T^{22}, y\right)
\end{array}\right.
$$

where $L_{3}$ and $L_{4}$ are known functions (note that $T^{12}$ is known) linear in $u_{1}, u_{2}, T^{11}, T^{22}$. At last, $u_{3}$ may be determined by (2.9). Finally, the complete problem is reduced to the system (7.2), (7.4) with the four unknowns $u_{1}, u_{2}, T^{11}, T^{22}$. As a whole it is a hyperbolic system with (double) characteristics the asymptotic curves. The system is diagonable and in fact it is writen in diagonal form. If the boundary conditions for $T^{11}, T^{22}$, allow us to solve the partial system (7.2), then (7.4) become a hyperbolic system for $u_{1}, u_{2}$. Then, according to the boundary conditions, $(7.2),(7.4)$ will be considered either as a system or as two systems to be solved successively.

Let us examine in this contex the model examples of section 3.

Example 3.1. All the boundary conditions concern u. It is not possible to solve (7.2) (7.4) in the context of classical theory of hyperbolic systems.

Example 3.2. $\Gamma_{1}$ is free, and the boundary conditions are

$$
T^{\alpha \beta} n_{\beta}=0 .
$$

As $T^{12}$ is known and the boundary is not tangent to the characteristics, $n_{1}$ and $n_{2}$ do not vanish on $\Gamma_{1}$ and $T^{11}, T^{22}$ are known there by (7.5). Thus, the partial system (7.2) may be solved, as we have a Cauchy problem for it. Then (7.4) is a hyperbolic system for $u_{1}, u_{2}$, which vanish on $\Gamma$. This gives again a Cauchy problem, and it may be solved uniquely.

Example 3.3. Analogous to the previous one, but in the second problem the data are given on characteristic curves (Goursat instead of Cauchy problem). 
Example 3.4. As Example 3.1; impossible to solve in the hyperbolic framework.

Example 3.5. Analogous to Example 3.3.

We then see that the variational method may be applied in some cases where the classical does not work. Nevertheless, we must emphasize that the structure of the space $\hat{V}$ is far from that of a classical elliptic boundary value problem. The structure of the solutions and in particular the behavior of $u_{3}$ shown at the end of section 6 should be compared with the structure of the «pseudo-bendings» in statics of hyperbolic shells (cf. [6] p. 248). Indeed it is known $[10,6]$ that for hyperbolic shells, zero belongs to the essential spectrum of the operator of the membrane approximation; the pseudobending are in fact Weyl's sequences showing that the inverse operator is not bounded.

Generally speaking the lack of rigidity of the hyperbolic shell in the membrane approximation with respect to shear implies that all the corresponding rigidity must be furnished by flexion terms. This fact has probably consequences with respect to stability and buckling.

\section{References}

[1] M. Bernadou and M. Ciarlet; Sur l'ellipticité du modêle linéaire de coques de W. T. Koiter in Computing Methods in Sciences and Engineering, R. Glowinski and J. L. Lions eds., p. 99-136, Lecture Notes in Econom. and Math. Systems (1976), 134, Springer.

[2] N. BourbaKI: Eléments de Mathématique, Intégration XIII, Hermann, Paris (1965).

[3] P. G. Ciarlet et B. Miara: Une démonstration simple de l'ellipticité des modèles de coques de W. T. Koiter et P. M. Nagdhi, Compt. Rend. Acad. Sc. Paris, série I (1991), p. 411-415.

[4] R. Courant and D. HilberT: Methods of mathematical physics, vol, 2, Interscience, New York (1962).

[5] A. L. Goldenveizer: Theory of elastic thin shells, Pergamon, New York (1961).

[6] A. L. Goldenveizer, V. B. Lidskil and P. E. Tovstik: Free vibrations of thin elastic shells, (in russian), Nauka, Moscow (1979).

[7] J. L. Lions: Equations différentielles opérationnelles et problèmes aux limites, Springer, Berlin, (1961). 
[8] F. I. Niordson: Shell theory, North-Holland, Amsterdam (1985).

[9] E. SánCheZ-PAlenCia: Statique et dynamique des coques minces, I: Cas de flexion pure non inhibée. Compt. Rend. Acad. Sc. París, série I, 309 (1989), p. 411-417.

[10] E. SÁnCHEZ-PALENCIA: Statique et dynamique des coques minces, II: Cas de flexion pure inhibée. Approximation membranaire, Compt. Rend. Acad. Sc. Paris, série I, 309 (1989), p. 531-537.

[11] E. SÁnCHeZ-PALENCIA: Passage à la limite de l'élasticité tridimensionnelle à la théorie asymptotique des coques minces, Compt. Rend. Acad. Sc. Paris, série II, 311 (1990), p. 909-916.

[12] E. SÁNCHEZ-PALENCIA: Asymptotic and spectral properties of a class of singular-stiff problems, Jour. Math. Pures Appl., 71 (1992), p. 379-406.

[13] V. I. SMIRnov: Course of higher mathematics, vol. 5, Pergamon, Oxford (1964).

This work is part of the project «Junctions in Elastic Multi-structures» of the program SCIENCE of the Commission of the European Communities (contract n. $\left.{ }^{\circ} \mathrm{SC} 1 * 0473-\mathrm{C}(\mathrm{EDB})\right)$. 